\title{
SmartActions: Context-Aware Mobile Phone Shortcuts
}

\author{
Akos Vetek, John A. Flanagan, Ashley Colley, and Tuomas Keränen \\ Nokia, P.O. Box 407, FIN-00045, Nokia Group, Helsinki, Finland \\ \{akos.vetek, adrian.flanagan, ashley.colley, \\ tuomas.j.keranen\} @nokia.com
}

\begin{abstract}
Mobile phones are often regarded as difficult to use due to their size restrictions. To improve on this, in this paper we described our approach using unsupervised learning to automate common tasks on a mobile phone, thereby requiring less key presses, by means of context-dependent quick-access shortcuts presented in the homescreen of the phone. We also briefly reviewed some of our user study findings, and raised the issue of possible privacy concerns with our implementation.
\end{abstract}

Keywords: Mobile interfaces, adaptive interfaces, context-dependent systems.

\section{Introduction}

Mobile phones have become ubiquitous and have now reached a stage where especially smartphones can provide users with a wide range of applications and services. Despite this, mobile phones are still often regarded as being difficult to use [1]. Due to the size restrictions of mobile phones, providing a more usable interface is hard to achieve. Context awareness can be used to facilitate the interaction of the user by recognizing the user context and determining and reacting to the user's needs in a given context by, for instance making available appropriate shortcuts, thus minimizing the user's interaction with the device through the UI.

Related work has focused on command-line prediction in a UNIX environment using a probabilistic bigram model to predict a user's next command [2] and also complete user commands [3]. More recently, Bridle and McCreath [4] investigated several approaches to induce shortcuts to automate making voice calls and sending text messages on a mobile phone. They found that some relatively simple approaches (e.g. most frequent commands) saved a considerable number of key presses, however such approaches presented the users with shortcuts that varied frequently. This is important as, in terms of usability, the aspect important in mobile phone design is not only efficiency (e.g. number of key presses) but predictability as well [5].

In this paper we describe our approach of inducing and applying both simple and complete shortcuts that automate a sequence of keypad presses. Our approach is based on context recognition, using an efficient unsupervised learning algorithm that requires no intervention from the user. The context-dependent shortcuts are displayed as a quickly glanceable list on the homescreen of the phone. 


\section{SmartActions Application}

An intelligent mobile device should not only know what its user wants, but also where and when its user wants it. Such a device should learn how it is used, that the user sets the alarm every night before bed, that typically she quickly checks her e-mail before leaving for the office in the morning and maybe even sends a text message to her spouse in the evening before leaving the office.

To this end, SmartActions is an implementation of a context recognition algorithm on the Nokia N80, a $3^{\text {rd }}$ edition S60 smartphone, driving an adaptive menu of lists of shortcuts. The list of items displayed in the menu adapts based on the current, recognized, user context and the applications associated with that context. The association between the context and the actions is learned from the user's previous use of applications in the same context. The context recognition is done using the K-Symbol String Clustering Map (K-SCM) [6], an efficient unsupervised clustering algorithm.

In the following two sub-sections the user interface and the operation of the KSCM algorithm underlying the application will be described in more detail.

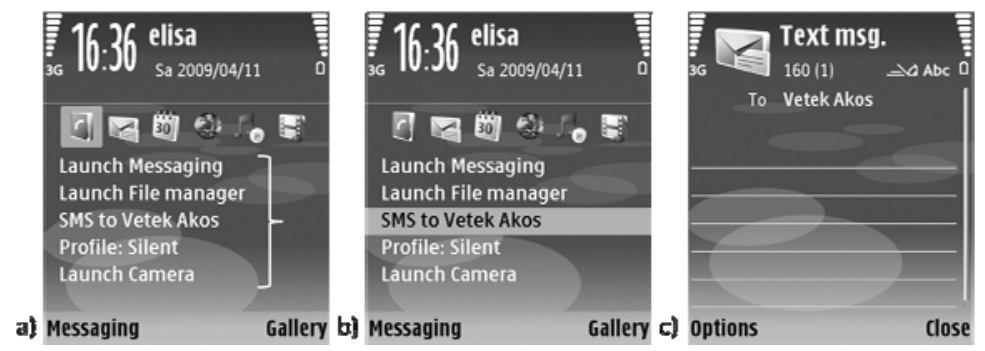

Fig. 1. a) A typical homescreen with SmartActions indicated by the brace. b) One of the shortcuts highlighted. c) The result of applying the shortcut with sender information filled in.

\subsection{User Interface}

The homescreen of $3^{\text {rd }}$ edition S60 devices can be customized via plug-ins. Plug-ins can provide an overview of the current state and contents of the device, such as the list of upcoming calendar appointments or a summary of new email messages.

SmartActions makes use of this framework and presents a quickly glanceable list of the 5 most likely functions the user will use in the current context (as defined by time and location) embedded in the homescreen (Fig. 1). The list includes not only application launcher shortcuts, such as "Launch Messaging", but also real user-level actions, such as "Call to Peter", "(send) SMS to Mary" or "(set) Profile: Silent". A more complete list of the currently implemented shortcuts is presented in Table 1. These suggested actions can be selected in a single key press, typically saving around 5 key presses or more. Should none of the displayed items be appropriate, the user can initiate the intended functionality at no loss in efficiency, as usual via the S60 application grid, as the rest of the phone's UI is left untouched. 
Table 1. List of possible SmartActions that can be learned with examples in brackets

List of possible SmartActions and examples
- Application launch (e.g. "Launch File Manager")
- Setting alarm ("Set alarm")
- Profile activation (e.g. "Profile: Silent")
- Establishing voice call to specified contact (e.g. "Call to John Doe")
- Sending SMS/MMS/email to specified contact (e.g. "SMS to John Doe")
- Opening browser with specified URL (e.g. "www.ovi.mobi")

\subsection{Context Recognition and Shortcut Association Using K-SCM}

An important challenge mobile phones provide for a learning system is the limited memory and processing power. This is especially crucial as any such approach must collect data and make predictions in an on-line and real-time manner.

With this in mind, we selected a modified version of the Symbol String Clustering Map (SCM), an algorithm for the unsupervised clustering of symbol string data based on adaptive learning [7]. The K-SCM algorithm improves on the SCM by requiring less memory and computational resources while giving the same result as the SCM [6]. The big benefit of unsupervised learning, as opposed to, for instance, ontologybased approaches, is that it requires no intervention from the user, and as such there is no need for any user settings at all.

As implemented in SmartActions, the inputs to the algorithm are: the location obtained from the GSM Cell ID parameters and the time and date encoded as symbol strings. The purpose of SmartActions is to learn different user contexts, in this case the context in terms of time and location, and to associate different applications that the user normally uses in these recognized contexts with the context. For example, if typically in the morning, at home, before leaving for work the user checks the news using the browser on the phone, SmartActions in this case would learn the context, "at home in the morning", with the location "home" determined from the GSM Cell ID, and the time "morning" from the clock. After several mornings the user would notice "Launch Browser" as one of the shortcuts on the homescreen, because the application has first of all learned the context "at home in the morning" and second of all learned to associate the use of the browser with this context. As the application is event driven, it does not learn all possible contexts, rather only those contexts where the user launches an application or otherwise interacts with the phone. As another example, at around "midday when at work" the user normally sends a text message to her spouse. This would correspond to a second context, SmartActions would learn the "midday when at work" context and associate the messaging application with this context. Hence around midday at work the messaging application would feature at the top of the list of suggested shortcuts in the homescreen.

In summary, the K-SCM algorithm is used so that contexts where the user interacts with the device are learned and those interactions are associated with the learned contexts. 


\section{Initial User Study Findings}

Twelve participants (6 male) used the application for a period of 2.5-3 weeks. Data was collected through focus group interviews, questionnaires and a post-interview. Initial results indicate that users generally liked the idea of a learning application (5.3 on a scale 1-6, 6 being most positive) and found it easy to understand how SmartActions functioned (4.3/6), however they expressed privacy concerns as the list of shortcuts was deemed too visible in the case of "confidential" contacts. Even though our aim was to design a quickly accessible, completely automatic system without any user settings, this is prompting us to rethink the appropriateness of displaying such potentially confidential shortcuts on the homescreen in an always on manner, or to introduce some means of customization to allow the permanent removal of unwanted shortcuts. In general, finding the appropriate way to display the results of the learning algorithm to the user is critical in the success of such systems.

\section{Conclusion and Future Work}

We explored the use of unsupervised learning to automate common tasks on a mobile phone, by means of context-dependent quick-access shortcuts displayed in the homescreen of the device. We also presented initial user study results that indicated that the appropriate way to display the results of the learning algorithm to the user is critical in the success of such systems. Experimenting with alternative ways of presenting the shortcuts, along with extending the set of context parameters and studying their effects will form the core of future work.

\section{References}

1. Amant, R.S., Horton, T.E., Ritter, F.E.: Model-based evaluation of cell phone menu interaction. In: Proceedings of the SIGCHI conference on Human factors in computing systems, pp. 343-350 (2004)

2. Davison, B.D., Hirsh, H.: Probabilistic Online Action Prediction. In: Proceedings of the AAAI Spring Symposium on Intelligent Environments, pp. 148-154 (1998)

3. Korvemaker, B., Greiner, R.: Predicting Unix command lines: adjusting to user patterns. In: Proceedings of the Seventeenth National Conference on Artificial Intelligence, pp. 230-235 (2000)

4. Bridle, R., McCreath, E.: Inducing shortcuts on a mobile phone interface. In: Proceedings of the 11th international conference on Intelligent user interfaces, pp. 327-329 (2006)

5. Lindholm, C., Keinonen, T.: Mobile Usability: How Nokia Changed the Face of the Cellular Phone. McGraw-Hill, Inc., New York (2003)

6. Flanagan, J.A.: Unsupervised Clustering of Context Data and Learning User Requirements for a Mobile Device. Modeling and Using Context, 155-168 (2005)

7. Flanagan, J.A.: Unsupervised clustering of symbol strings. In: Proceedings of the International Joint Conference on Neural Networks, pp. 3250-3255 (2003) 